Tema: Logística de Implantação de Novas Plantas e Expansões dos Parques Industriais

\title{
OTIMIZAÇÃO DO TRANSPORTE FERROVIÁRIO OUTBOUND NA ARCELORMITTAL MONLEVADE*
}

\author{
Paulo Eduardo Melo da Cunha ${ }^{1}$ \\ llemar Geraldo de Vasconcelos Júnior ${ }^{2}$ \\ Eduardo de Oliveira Diniz ${ }^{3}$ \\ André Luiz Santana Lea/ $\left.\right|^{4}$ \\ Virgílio Reis ${ }^{5}$ \\ Ferdinando Mario Giudice ${ }^{6}$ \\ André Pessoa Paulocci ${ }^{7}$
}

\section{Resumo}

Com um cenário de constante mudança na área e logística, uma demanda cada vez maior por transporte rápido, eficiente e a baixo custo, muito se tem exigido dos gestores de logística na solução, otimização e implementação de soluções criativas para desatar o nó logístico existente hoje no país. A falta de infra-estrutura tanto interna, quanto externa tem dificultado o trabalho e comprometido os resultados globais das empresas. Muitos trabalhos já foram realizados, mas as constantes mudanças, aumento dos volumes transportados em cada modal, a falta de planejamento governamental, tem exigido novas formas de trabalhar. Um relacionamento mais próximo dos fornecedores de transporte, com grande envolvimento dos clientes e áreas comerciais é fundamental para os bons resultados e é desta forma que buscamos otimizar o fluxo ferroviário para a região da grande $\mathrm{BH}$.

Palavras-chave: Transporte intermodal; Operador logístico; Melhoria de confiabilidade.

\section{OPTIMIZATION OF OUTBOUND RAIL SHIPMENTS AT ARCELORMITTAL MONLEVADE}

\section{Abstract}

With a challanging cenario, were there is a need for a quicker, more efficient and low cost of transportation mode, much has been demanded from the Logistic managers, on finding creative solutions for the logistic caos installed in our country these days. The lack of infra-structure, internally or externally, have been impacting on companies results. Many solutions were implemented, but the constant changes, increase in volumes transported in every mode, lack of government planning, have demanded new actions. A closer relationship among transportation suppliers and customers, and commercial staff is crucial to boost up results, and is how we expect to optimize the rail transportation mode to the Great Belo Horizonte region.

Keywords: Third party logistics; Inter-modal transportation; Reliability improvement.

1 Engenheiro Metalurgista, Mestrando FDC, PUC, Consultor de Logística, ArcelorMittal Longos, João Monlevade, MG, Brasil.

2 Bacharel em Administração de Empresas, Analista de Logística, ArcelorMittal Longos, João Monlevade, MG, Brasil.

3 Engenheiro Metalurgista, Mestre em Metalurgia, Gerente de Logística, ArcelorMittal Longos, João Monlevade, MG, Brasil.

4 Administrador de Empresas, Gerente Geral Siderurgia, VLI, Belo Horizonte, MG, Brasil.

5 Engenheiro de Produção, Gerente Comercial, VLI, Belo Horizonte, MG, Brasil.

6 Engenheiro Mecânico, Mestrando em Logística FUMEC, Especialista de Logística, ArcelorMittal Longos, Belo Horizonte, Minas Gerais, Brasil.

7 Engenheiro Metalurgista, Gerente de Logística, Belgo Bekaert Arames, Contagem, Minas Gerais, Brasil.

\footnotetext{
* Contribuição técnica ao 33० Seminário de Logística - Suprimentos, PCP, Transportes, 13 a 16 de maio de 2014, São Paulo, SP, Brasil.
} 


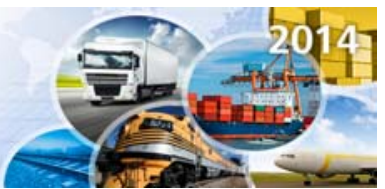

\section{INTRODUÇÃO}

Muito se tem feito com o objetivo de otimizar, melhorar o atendimento a clientes e reduzir custos das atividades logísticas. Trabalhos anteriores apresentados neste seminário mostram uma grande preocupação com a infraestrutura e a demanda da região Metropolitana de Belo Horizonte. O constante crescimento dos volumes transportados nos diversos fluxos, sem um investimento e planejamento adequados que suporte este crescimento, tem gerado inúmeros gargalos, podendo inviabilizar projetos, como a dificuldade de atendimento ao projeto anteriormente desenvolvido. No caso da ArcelorMittal Monlevade, o atendimento à Belgo Bekaert Contagem era feito através do envio ferroviário de Monlevade ao pátio da Tora Logística em Eldorado. A Tora fazia o gerenciamento do estoque em conjunto com a Belgo Bekaert e enviava o material de rodovia até Contagem [1].

O fluxo ferroviário para atendimento à região da grande $\mathrm{BH}$ (região denominada Parque Industrial) foi prejudicado em função da grande demanda de transporte nesta região com concorrência de produtos de diferentes segmentos, gerando alto tempo de lead time comprometendo a eficácia do sistema. Dessa forma não conseguimos atingir a meta prevista de 36 horas.

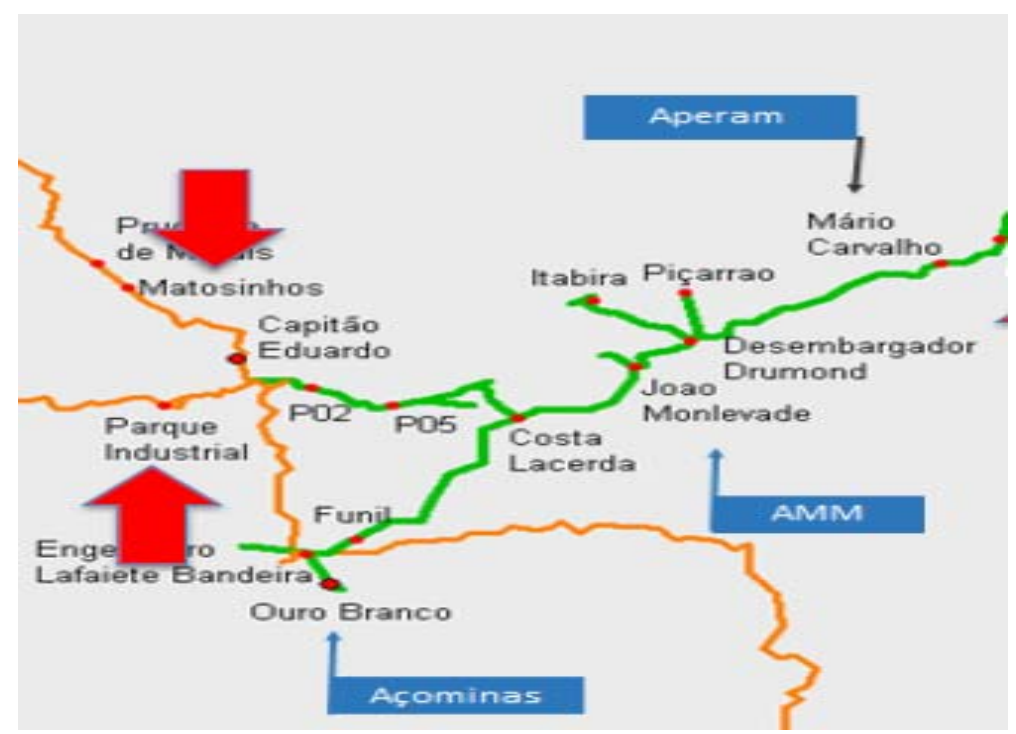

Figura 1. Fluxo ferroviário região grande BH.

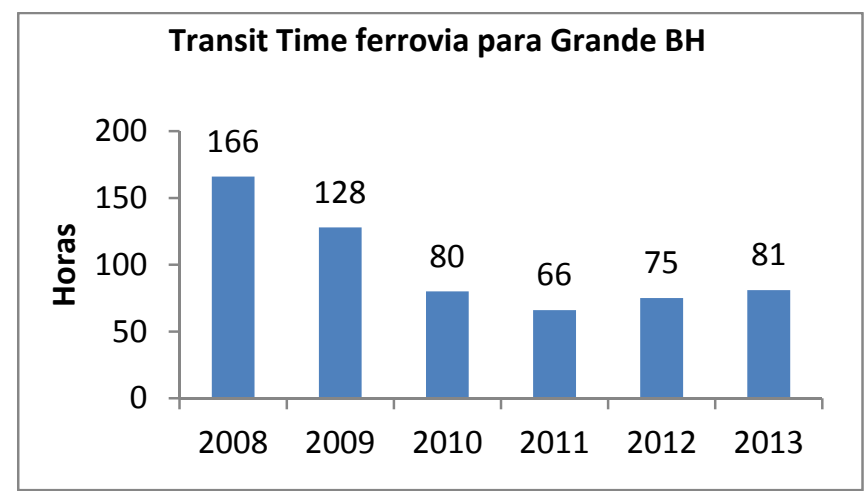

Figura 2. Transit time ferrovia para grande $\mathrm{BH}$.

\footnotetext{
* Contribuição técnica ao $33^{\circ}$ Seminário de Logística - Suprimentos, PCP, Transportes, 13 a 16 de maio de 2014, São Paulo, SP, Brasil.
} 


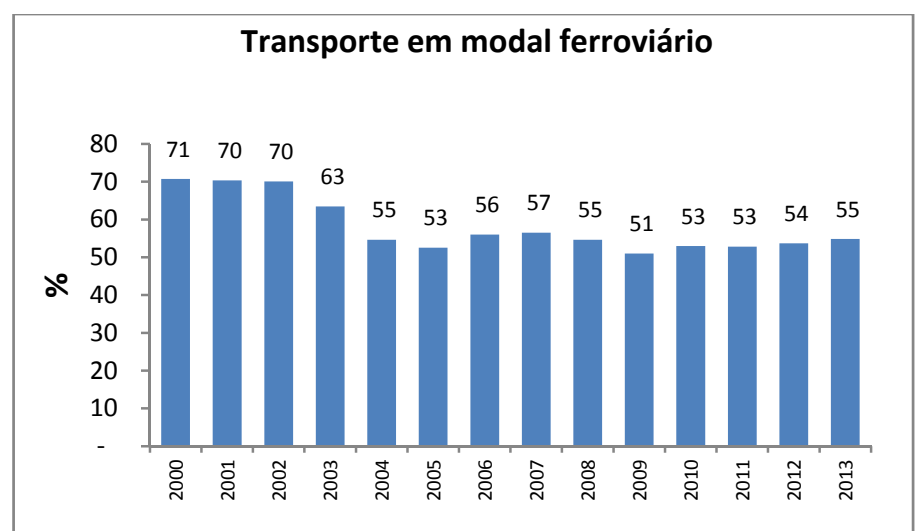

Figura 3. Transporte em modal ferroviário.

O problema descrito tende a se agravar devido à intenção do Governo Estadual de incrementar o transporte ferroviário para passageiros, o que limitará mais ainda o fluxo da área citada, aliado também com a possibilidade de início das obras de duplicação da BR 381, que limitará o transporte rodoviário na região, exigindo uma maior utilização do modal ferroviário.

A retomada da expansão de Monlevade, com a duplicação da expedição prevista para início de 2015, demandará mais ainda deste sistema, pois teremos uma sobreposição de gargalos, da estocagem interna, transporte e recepção no cliente final, exigindo mudanças e um novo desenho fluxos para suportar este crescimento.

Neste contexto a ArcelorMittal (embarcadora), VLI (Transportadora) e Belgo Bekaert (cliente) se uniram na busca de uma solução que atendesse a todas as necessidades, no curto prazo e nas demandas futuras

\section{MATERIAIS E MÉTODOS}

Foi realizado um estudo completo de todo o fluxo, considerando desde o estoque inicial de fio máquina, passando pelo carregamento, transporte, descarga e estocagem no cliente final [2], constatando-se os seguintes problemas:

- Falta de capacidade de estocagem após produção na usina de Monlevade, item que se tronará crítico com a duplicação da produção;

- Falta de vagões disponíveis para carregamento em função do congestionamento do fluxo, agravando mais o problema de estocagem na origem;

- Lead Time de transporte alto, comprometendo o atendimento ao cliente e disponibilização de vagões para carregamento;

- Utilização de "janelas" para circulação nas linhas do Parque Industrial, comprometendo a otimização da descarga;

- Dificuldade de atendimento ao cliente final, em função de produtos presos no circuito, sem possibilidades de retirada dos mesmos;

- Excesso de solicitações de urgências rodoviárias onerando o processo de atendimento;

- Falta de confiança no processo como um todo.

\footnotetext{
* Contribuição técnica ao $33^{\circ}$ Seminário de Logística - Suprimentos, PCP, Transportes, 13 a 16 de maio de 2014, São Paulo, SP, Brasil.
} 


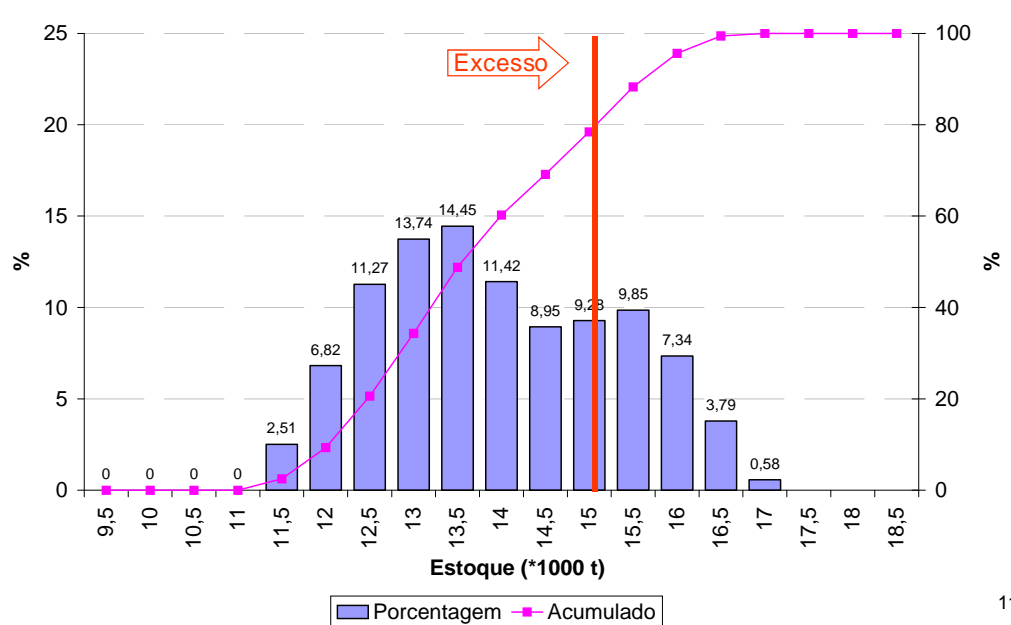

Figura 4. Projeção de estoque de Fio Máquina Usina Monlevade pós expansão.

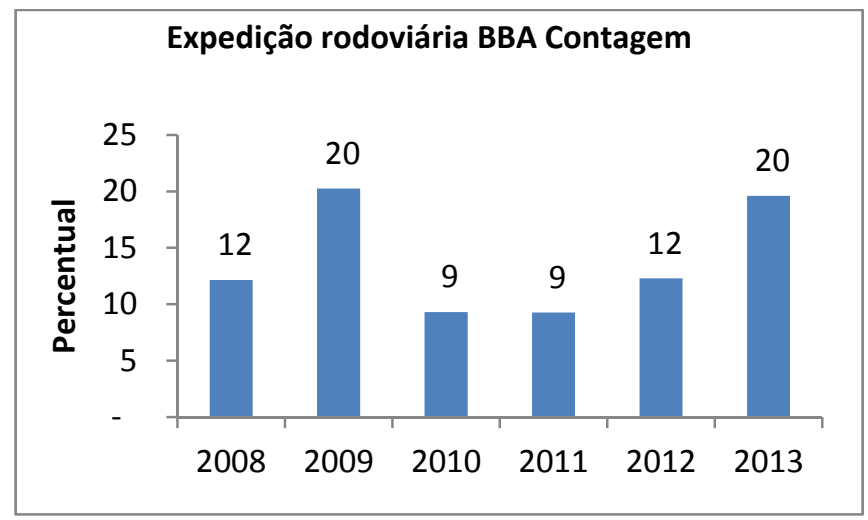

Figura 5. Expedição rodoviária para BBA Contagem.

Foi então criado um grupo de trabalho envolvendo todas as áreas (AMM, VLI, Vale, AMB e BBA), com intuito de achar uma solução conjunta que aumentasse a produtividade do sistema e suportasse a expansão futura das instalações de Monlevade.

Objetivos a curto prazo:

- Instalar um depósito avançado para estocagem de fio máquina e regulagem do fluxo, situado fora da zona de gargalo da região metropolitana de Belo Horizonte;

- Viabilizar o carregamento em vagões GFE na usina de Monlevade, para proporcionar o aproveitamento de todos os vagões recebidos com matérias primas;

- Desenvolver um desenho de trens diferenciado (trem unitário) com um lead time compatível com as necessidades do cliente;

- Criar um pulmão de estoque para manutenção do abastecimento e atendimento aos clientes finais da Belgo Bekaert Contagem;

- Desenvolver junto com o operador logístico atual o atendimento da ponta rodoviária (veículos rastreados e dedicados);

- Integrar todo o fluxo ferroviário eletronicamente (interface VLI/AM).

\footnotetext{
* Contribuição técnica ao $33^{\circ}$ Seminário de Logística - Suprimentos, PCP, Transportes, 13 a 16 de maio de 2014, São Paulo, SP, Brasil.
} 


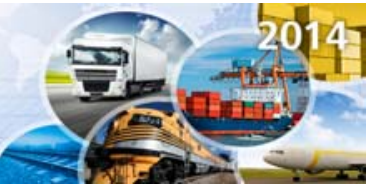

Objetivos a médio prazo:

- Otimizar os processos de carregamento e estocagem na usina de Monlevade através de desenvolvimento de sistemas inteligentes, para suportar as novas demandas e crescimento nos volumes de produção

- Interligar eletronicamente todo o processo de distribuição, através da instalação de uma central de operações em Monlevade interligada com o sistema da VLI.

- Ampliar a utilização do TISL com outros clientes, aliviando a utilização da BR 381

Preparar para futuras ampliações das instalações de acordo com a demanda do mercado.

\section{IMPLANTAÇÃO}

- Construção do terminal TISL (Terminal Integrador de Santa Luzia) pela VLI, para atendimento de demanda máxima siderúrgica de 140.000 t/mês (Chapas Grossas, Bobinas/Fardo, Bobinas Inox/ carbono e elétrico, Fio-máquina), fora da área congestionada da Grande $\mathrm{BH}$;

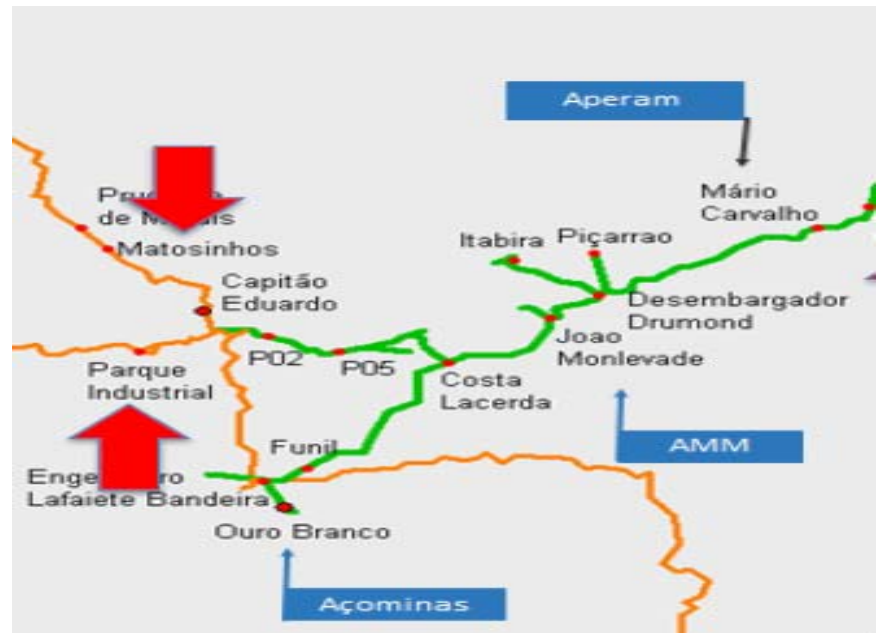

Figura 6. Localização do Terminal TISL.

- Área de operação útil com 3 linhas para posicionamento de vagões com capacidade para até 90 vagões posicionados;

- Trem padrão de 90 vagões;

- Pista de carregamento interno;

- 2 Postos de peação com total de 6 slots;

- 3 pórticos com capacidade de $25 \mathrm{t}$;

- 2 pontes rolantes com capacidade de 25 e $35 \mathrm{t}$.

- Definição dos processos de recepção, movimentação, estocagem e expedição em conjunto VLI e Belgo Bekaert Contagem, de modo a atender todas as especificações de serviço e qualidade do cliente;

- Definição de um modelo de trem com uma faixa de 40 a 50 vagões partindo de Monlevade com uma meta de 36 horas de lead time;

- Utilização da Central de Tráfego da ArcelorMittal para gerenciar a ponta rodoviária entre o TISL e Contagem, com os veículos sendo rastreados e monitorados.

\footnotetext{
* Contribuição técnica ao $33^{\circ}$ Seminário de Logística - Suprimentos, PCP, Transportes, 13 a 16 de maio de 2014, São Paulo, SP, Brasil.
} 

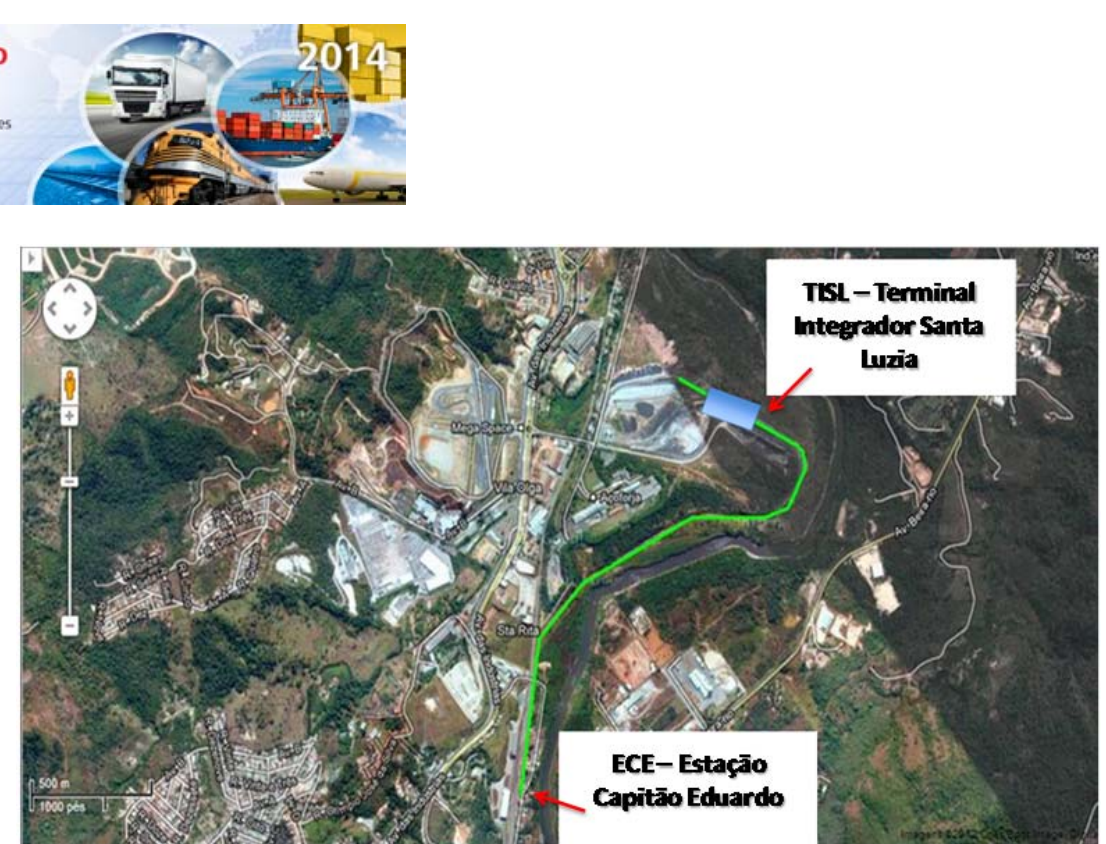

Figura 7. Ligação do TISL com estação de Capitão Eduardo.

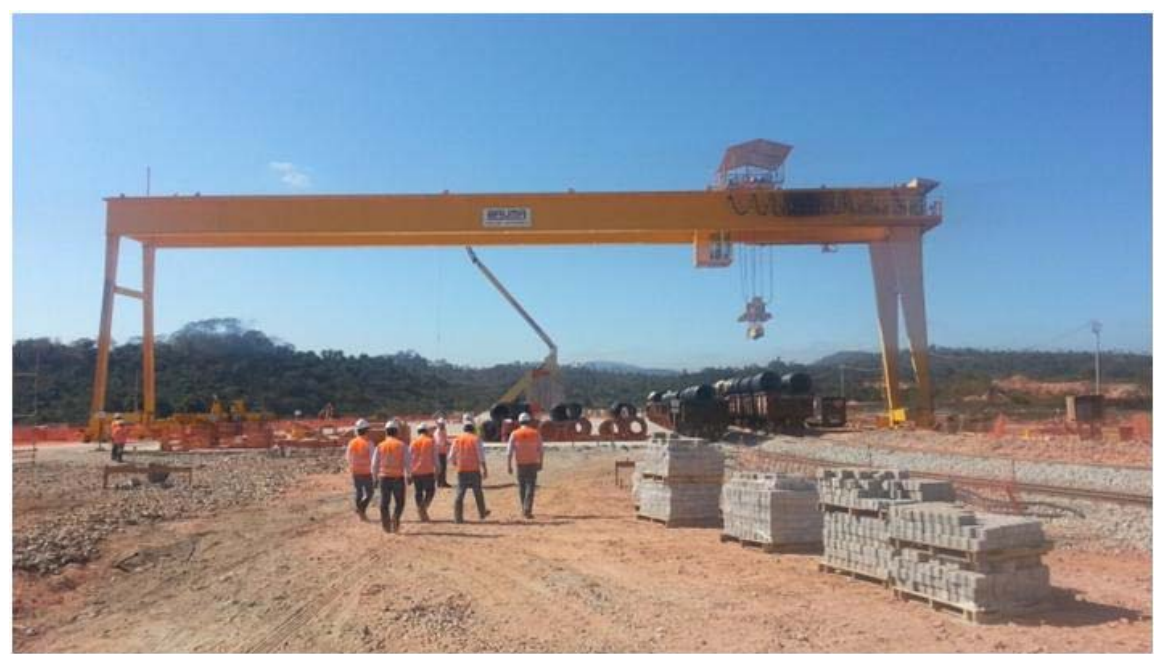

Figura 8. Construção do terminal TISL.

\section{RESULTADOS}

O terminal começou a ser operado a partir de Outubro 2013, a operação está em fase de maturação e ajustes dos processos entre as partes (estocagem, manuseio, carregamento e entrega).

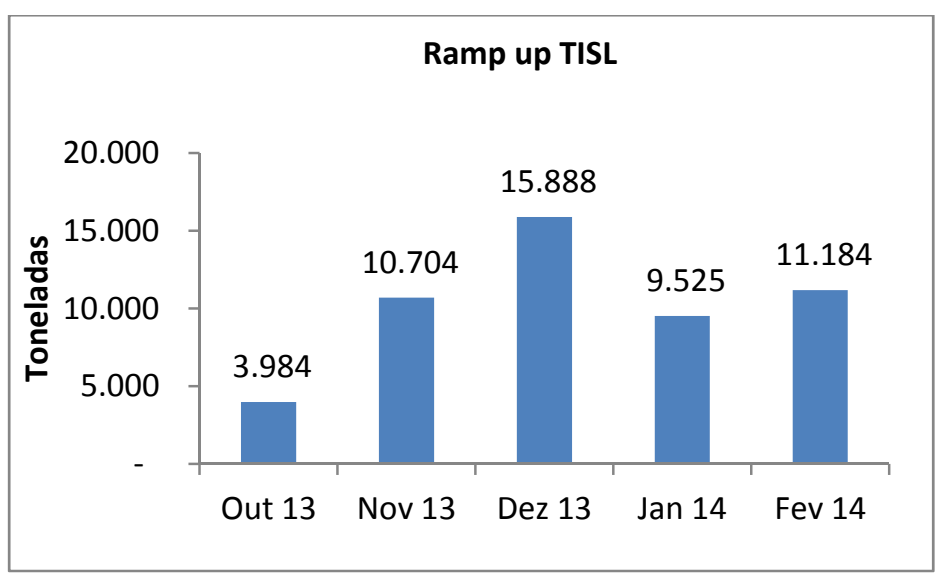

Figura 9. Ramp up utilização TISL.

\footnotetext{
* Contribuição técnica ao $33^{\circ}$ Seminário de Logística - Suprimentos, PCP, Transportes, 13 a 16 de
} maio de 2014, São Paulo, SP, Brasil. 

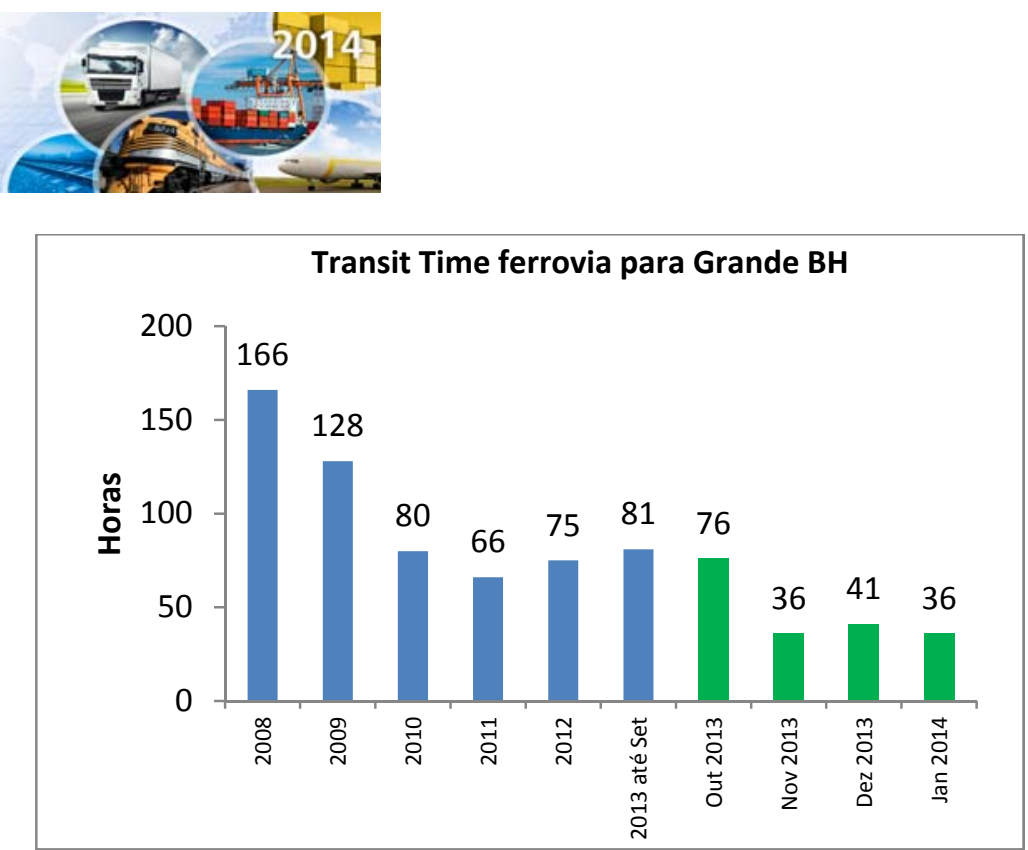

Figura 10. Transit time ferrovia para Grande BH.

Além dos resultados acima, a utilização do TISL proporcionou uma maior disponibilização de vagões em função do menor tempo de circulação.

O estoque em trânsito para a Belgo Bekaert Contagem também foi reduzido, com consequente redução do volume de solicitações de urgências rodoviárias.

\section{DESENVOLVIMENTOS EM ANDAMENTO}

- Estudo para ampliação do terminal em função da expansão da ArcelorMittal Monlevade

- Interligação eletrônica com a VLI para possibilitar a inclusão dos dados da ferrovia na Central de Operações da ArcelorMittal Monlevade

- Utilização do terminal para clientes de São Paulo (processo de cross docking)

- Implantação do novo sistema de WM da VLI

- Revisão da metodologia de estocagem em movimentação dos produtos (VLI/ BBA).

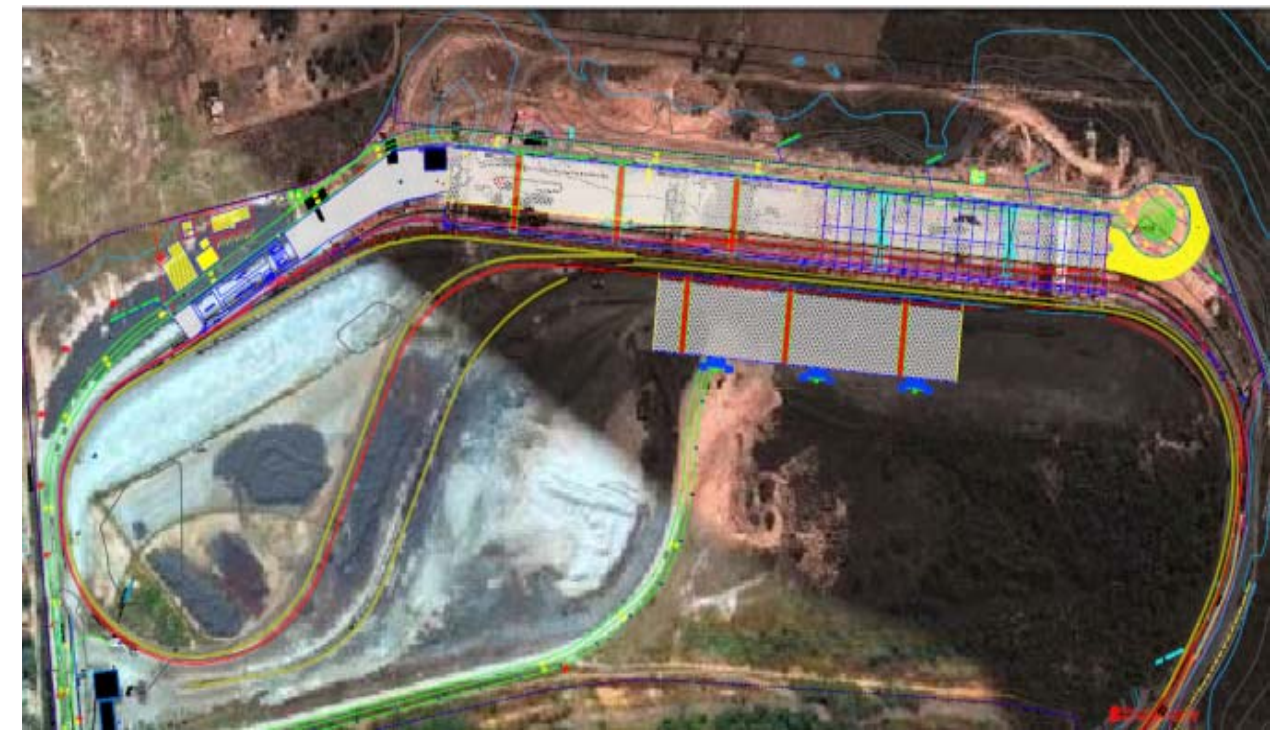

Figura 11. Estudo para ampliação do terminal TISL.

\footnotetext{
* Contribuição técnica ao 33o Seminário de Logística - Suprimentos, PCP, Transportes, 13 a 16 de
} maio de 2014, São Paulo, SP, Brasil. 


\section{CONCLUSÃO}

A característica dinâmica da Logística, aliada aos cenários de baixo planejamento governamental e mudanças nos mercados atuais exige uma constante readequação e aprimoramento dos fluxos existentes.

É fundamental a manutenção e forte envolvimento dos parceiros da cadeia de abastecimento de modo não fragmentar e isolar as partes que compõe o processo total.

\section{REFERÊNCIAS}

1 Cunha, PEM, Rajão CT, Medina M, Alvim A, Costa GD, Vasconcelos Jr. IG. Otimização do transporte ferroviário outbound na ArcelorMittal Monlevade. In: $29^{\circ}$ Seminário de Logística, Suprimentos, PCP, Transportes; 2010; Joinville, Brasil. São Paulo: ABM; 2010.

2 Cunha PEM, et al. Projeto empresarial [MBA]. Nova Lima: Fundação Dom Cabral; 2003.

* Contribuição técnica ao $33^{\circ}$ Seminário de Logística - Suprimentos, PCP, Transportes, 13 a 16 de maio de 2014, São Paulo, SP, Brasil. 